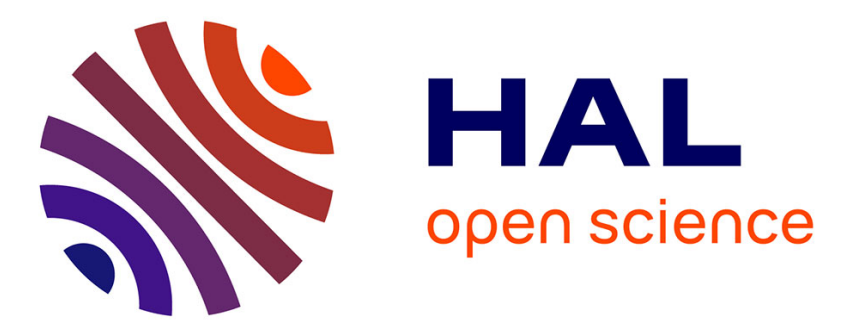

\title{
Arthrogryposis in children: Etiological assessments and preparation of a protocol for etiological investigations
}

E. Wallach, U. Walther- Louvier, C. Espil-Taris, Francois Rivier, E. Baudou, C. Cances

\section{- To cite this version:}

E. Wallach, U. Walther- Louvier, C. Espil-Taris, Francois Rivier, E. Baudou, et al.. Arthrogryposis in children: Etiological assessments and preparation of a protocol for etiological investigations. Archives de Pédiatrie, 2018, 10.1016/j.arcped.2018.05.004 hal-01820295

\section{HAL Id: hal-01820295 \\ https://hal.umontpellier.fr/hal-01820295}

Submitted on 24 Jan 2020

HAL is a multi-disciplinary open access archive for the deposit and dissemination of scientific research documents, whether they are published or not. The documents may come from teaching and research institutions in France or abroad, or from public or private research centers.
L'archive ouverte pluridisciplinaire HAL, est destinée au dépôt et à la diffusion de documents scientifiques de niveau recherche, publiés ou non, émanant des établissements d'enseignement et de recherche français ou étrangers, des laboratoires publics ou privés. 


\title{
Arthrogryposis in children: Etiological assessments and preparation of a protocol for etiological investigations
}

\author{
E. Wallach ${ }^{\mathrm{a}, \mathrm{b}, *}$, U. Walther- Louvier ${ }^{\mathrm{c}}$, C. Espil-Taris ${ }^{\mathrm{d}}$, F. Rivier ${ }^{\mathrm{c}}$, E. Baudou $^{\mathrm{a}}$, C. Cances $^{\mathrm{a}}$ \\ anité de neuropédiatrie (Neuropaediatrics Unit), hôpital des enfants, CHU Purpan, 330, avenue de Grande-Bretagne, 31059 Toulouse cedex 9, France \\ ${ }^{\mathrm{b}}$ Soins de suite et de réadaptation pédiatriques spécialisés (SSR) (Specialist pediatric follow-up and rehabilitation care), Centre Paul-Dottin, 26, avenue \\ Tolosane, 31522 Ramonville-Saint-Agne cedex, France \\ ${ }^{\mathrm{C}}$ Unité de neuropédiatrie (Neuropediatrics Unit), hôpital Gui-de-Chauliac, CHU de Montpellier, 191, avenue du Doyen-Gaston-Giraud, 34090 Montpellier, \\ France \\ ${ }^{\mathrm{d}}$ Unité de neuropédiatrie (Neuropediatrics Unit), groupe hospitalier Pellegrin, CHU de Bordeaux, place Amélie-Raba-Léon, 33000 Bordeaux, France
}

Keywords:

Arthrogryposis

Amyoplasia congenita

Neurological etiologies

Myopathies

Multidisciplinary

Genetic

Protocol

\begin{abstract}
A B S T R A C T
Introduction: Arthrogryposis is a descriptive term defining a sign. It describes a set of joint contractures, sometimes identifiable in utero, present from birth and nonprogressive. This term includes a heterogeneous group of diseases, of neurological, neuromuscular, genetic or mechanical origin. The common physiopathological mechanism is fetal immobility syndrome. Two types of classification have been developed: a clinical one (types I, II and III) and an etiological one. The main aim of this study was to define a standardized protocol for etiological investigation based on a descriptive analysis of the various etiologies identified in a population of children followed up for arthrogryposis. Its secondary aim was to assess first the comprehensiveness and relevance of the complementary assessment and second the way in which the classifications proposed by Professor Judith Goslin Hall are applied.

Material and methods: Retrospective multicenter observational study. We enrolled pediatric patients with arthrogryposis being treated at a reference center for neuromuscular diseases, i.e., in three university hospital pediatric neurology units, between February 1997 and January 2017.

Results: Forty-two patients ( 25 boys and 17 girls) were enrolled. According to the clinical classification (Hall et al.), this population consisted of eight cases of type 1 arthrogryposis (19.1\%), 14 type II (33.3\%) and 20 type III (47.6\%). The main etiology was neurological (19.1\%), predominantly involving problems with gyration of a polymicrogyria type. Myopathic origin accounted for $9.5 \%$ of the population, predominantly involving genotyped distal arthrogryposis (ECEL1 gene). Additional tests produced a diagnosis of $25 \%$ type I, $43 \%$ type II and $75 \%$ type III.

Conclusion: Arthrogryposis is a sign suggesting multiple etiologies. The main ones are neurological. Several genes have recently been identified, explaining the physiopathological mechanisms. The diagnostic process must be rigorous and coordinated within a multidisciplinary team, following a shared protocol for analysis.
\end{abstract}

\section{Introduction}

The word "arthrogryposis" means joint ("arthro") and curved ("gryp"). This is a descriptive term defining a sign. It is used to describe a set of contractures (articular) in different parts of the body, mainly involving the limbs, as well as functional limitation

\footnotetext{
* Corresponding author. Unité de Neuropédiatrie (Neuropediatrics Unit), hôpital des enfants, 330, avenue de Grande-Bretagne, TSA 70034, 31059 Toulouse cedex 9, France.

E-mail addresses: E.Wallach ${ }^{a{ }^{a}}{ }$ wallach.e@chu-toulouse.fr, "aut0005">E.Wallach ${ }^{\mathrm{ab}_{*}}$ wallach.e@chu-toulouse.fr (E. Wallach)
}

affecting several movements. This term includes a heterogeneous group of diseases (muscular, genetic, neurological) and must be used if the contractions are present at birth, are nonprogressive, and involve more than one limb [1]. Arthrogryposis remains a general term, found in different formulae, which at times may be confusing.

Arthrogryposis multiplex congenita is characterized by congenital, nonprogressive and symmetric joint contractures that involve at least two different body areas. Both upper and lower limbs are usually involved with skin disorders and characteristic facies. This group includes amyoplasia congenita, distal arthrogryposis and arthrogryposis syndromes. At the moment, the term "multiple congenital contractures" (MCC) is used [2-4]. 
Fetal immobility syndrome (FIS) is a shared mechanism at the origin of arthrogryposis. It defines a set of deformations resulting from the reduction or elimination of active fetal movements. It combines, in a more or less complete way, craniofacial dysmorphology, pulmonary hypoplasia, slightness, osseous deformation, skin disorders (edematous infiltration, protrusion, grip) and a short neck. This syndrome was called "Pena Shokeir" in the 1970s and was thought to be a malformative sequence of fetal akinesia. The occurrence of this sign is rare, on the order of $1 / 3000$ to $1 / 5000$ live births [2]. Its prognosis and severity depend on how early this sequence appears.

Studying arthrogryposis leads indirectly to an embryological analysis of movement in utero. The initial physiopathological process is the reduction of active fetal movements and/or fetal immobility corresponding to fetal hypokinesia (or fetal akinesia) leading to varying degrees of secondary deformation. Other associated problems are micrognathia, in utero growth restriction (IUGR), short limbs, pulmonary hypoplasia or a short small intestine, and pterygium. The reduction in active fetal movements involves numerous paths of development and therefore various etiologies. There is no specific anatomopathological lesion for a given etiology, with numerous factors responsible for stiffness of the joints (fibrosis of ligaments, capsular fibrosis, contracture of muscles around the joints).

Over 400 specific pathologies associated with arthrogryposis have been described and over one-third of these are of genetic origin. Hall and Kiefer have listed 320 genes and arranged them in 22 pathways. Two-thirds of etiological diagnoses of arthrogryposis can be made before the age of 2 years [5,6]. Etiologies can be divided into two categories:

- secondary to a problem with the fetal environment (oligoamnios, multiple pregnancy, compression by a uterine or adnexa mass, maternal exposure (curare, fetal antireceptor antibodies to acetylcholine by maternal illness);

- associated with an intrinsic problem with fetal development.

Within this category, a distinction can be made between:

- primitive muscular disorders. The myopathic process is characterized by a problem with muscle structure or function leading to a reduction in fetal movement. If this process affects fast contraction muscles, it is responsible for distal arthrogryposis. The muscle damage corresponds to $20-30 \%$ of cases and includes, in particular, amyoplasia congenita (muscle that has formed but cannot develop its properties) or a fatal form of multiple pterygium syndrome. The other pathologies involved are congenital muscular dystrophies, Steinert myotonic dystrophy syndrome, congenital myopathies identified at a prenatal stage, and mitochondrial or metabolic disorders. The severe forms may be responsible for MCC;

- fetal akinesia of neurogenic origin is the most common MCC (70-80\%) [7]. The neuropathic process includes: muscle problems acquired through denervation of central (spinal cord degeneration with depletion of motoneurons in the anterior horn and motor nuclei or infantile spinal amyotrophy) or peripheral origin (abnormality of the neuromuscular junction); the neurological process includes anomalies in neuronal migration, other abnormalities involving malformation, or degeneration of the pyramidal cells);

- problems with conjunctival dermoid development (genodermatoses, osteochondrodysplasia).

The diagnostic process initially involves an obstetric, perinatal and family assessment. The complete clinical examination must and family assessment. The complete clinical examination must of view. The assessment of joint damage and the estimation of the degree of flexion or extension may be completed by taking photographs, valuable references for ensuring follow-up of the patient's development [6]. Natural development and the response to rehabilitation treatment may sometimes help establish the etiological diagnosis.

The aim of the proposed classifications is to better understand the physiopathological mechanism and to facilitate the diagnostic and therapeutic processes. In 1981, Prof. Hall suggested a pragmatic clinical approach to arthrogryposis, in three categories:

- type I: only involving the limbs;

- type II: involvement of the limbs and other areas of the body or systems;

- type III: malfunction of the central nervous system or associated cognitive impairment $[1,8]$.

In 2009, Bamshad et al. revisited the classification, etiologies and treatment of arthrogryposis [9]. The Bamshad classification concerns distal arthrogryposis in particular. It is a simplified classification with clearer diagnostic criteria and is easier to use. Treatment must be multidisciplinary and coordinated. It is preventive, sometimes curative and palliative.

The main aim of the study presented herein was to define a protocol for etiological analysis that is standardized following a descriptive analysis of the various etiologies identified in a population of children followed up for arthrogryposis in a reference center for neuromuscular diseases in the GrandSud-Ouest in France. The secondary aims were first to assess the comprehensiveness and relevance of the additional check and second the application of the classifications proposed by Professor Judith Goslin Hall.

\section{Material and methods}

This study was a retrospective multicenter observational study. We included pediatric patients with arthrogryposis receiving treatment at the Grand-Sud-Ouest reference center for neuromuscular diseases, i.e., in the Bordeaux, Montpellier and Toulouse University Hospital pediatric neurology units, between January 1997 and December 2016. The enrollment criteria were: impairment of more than two joints, defined as arthrogryposis. The exclusion criteria were: isolated cases of club foot, fewer than two impaired joints, and prenatal diagnoses with a decision to terminate the pregnancy for medical reasons.

The data collected involved the progress of the pregnancy (taking drugs, chronic infection or illness), prenatal ultrasound tests (assessment of active fetal movements, quantity of amniotic fluid); the delivery and perinatal data; family history (consanguinity, myasthenia gravis, Steinert myotonic dystrophy, other cases presenting arthrogryposis); the impaired joints at diagnosis; the associated signs; the complementary assessments carried out and their results; clinical course and treatment.

Medical data were gathered between January and March 2017. Since the study was retrospective, there was no need to request ethics committee approval (JORF10/05/2017). A declaration was made to the CNIL (French Data Protection Authority, No. 2092320).

\section{Results}

Forty-two patients were enrolled: 35 from the Occitane region and seven from Aquitaine. Our sample consisted of 25 boys and 17 girls, including two siblings. Eleven patients came from a family 


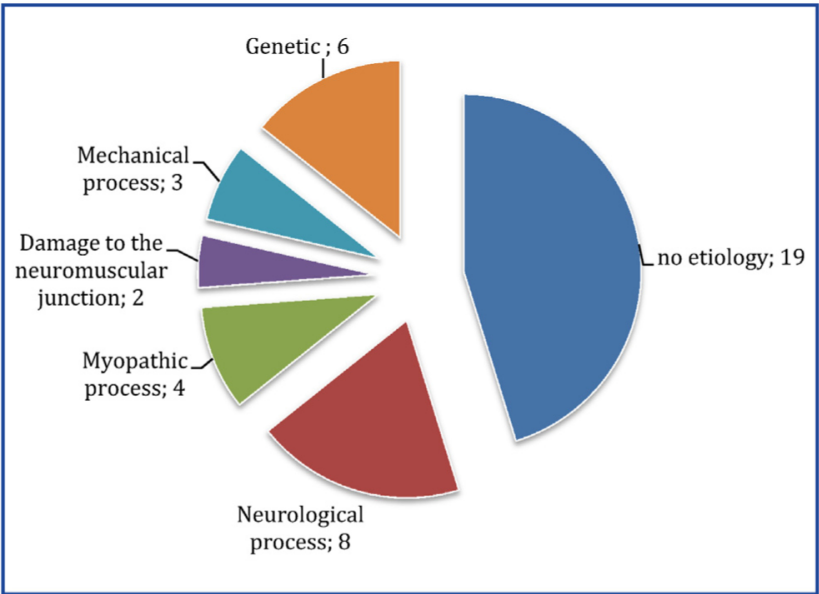

Fig. 1. Diagram of the various etiologies of arthrogryposis in the study population. NMJ: neuromuscular junction.

According to the Hall classification the clinical characteristics were: 20 type III (47.6\%), 14 type II (33.3\%) and eight type I (19.1\%).

When gathering the data, the etiological diagnosis had been made in 23 patients (55\%). The etiologies were divided up as follows (Fig. 1):

- neurological process: eight patients (19.1\%). Four patients had polymicrogyria, one had extensive cerebral ischemic lesions in the posterior regions, and one had an anoxic-ischemic lesion of the cervical and thoracic regions of the spinal cord. Two cases involved impairment of the anterior horn of the spinal cord;

- syndromic genetic etiologies: six patients (14.3\%). Three children from the same family presented with Crisponi syndrome. One patient had partial trisomy $4 \mathrm{p}$ associated with partial monosomy 18q, and one patient had Van Den Ende Gupta syndrome. Finally, one patient had a 49XXXXY chromosomal aberration;

- environmental cause: three patients (7.1\%), as a result of a septate bicornuate uterus or prolonged rupture of the membranes;

- myopathic process: four patients (9.5\%). Three patients had an identified ECEL1 mutation. One patient had a MYH3 mutation;

- impairment of the neuromuscular junction: two patients (4.8\%). One patient presented with a congenital myasthenic syndrome, the second a genotyped Escobar syndrome.

The complementary assessments were carried out after an initial clinical evaluation and in accordance with the etiological hypothesis or hypotheses formulated during the course of the disease. Only a few patients benefited from a comprehensive review exploring all the possible etiological avenues. The breakdown of the complementary assessments according to clinical category is shown in Table 1 .

\section{Discussion}

Our series consists of 42 cases over 20 years diagnosed and followed up in the Grand Sud-Ouest reference center for neuromuscular diseases. Twenty cases were followed up at the Toulouse University Hospital, 15 at the Montpellier University Hospital and seven at the Bordeaux University Hospital. These disparities are due to selection bias, since not all the patients were referred to the various reference center sites and therefore were not listed in the CEMARA shared database. This recruitment bias, reinforced by the retrospective nature of the study, mainly limits the evaluation of the prevalence of arthrogryposis in this population.

The population mainly consisted of boys (60\%) with a sex ratio of 1.5, in line with documented data. Type III arthrogryposis was predominant with 20 cases (47.6\%), then 14 cases (33.3\%) of type II and eight cases of type I (19.1\%). In the Eurocat study, $37 \%$ of patients were classified as having type I arthrogryposis, $51 \%$ as having type II and $12 \%$ as having type III (with chromosomal abnormalities predominating) [10]. In the Lowry et al. series, grouping 103 cases of arthrogryposis, 34\% were type I, 34\% type II and $31 \%$ type III [2]. The Hall classification provides a pragmatic clinical approach and thus guides the diagnostic process for this complex sign. It is useful for comparing the various populations, medical teams and their practices. The predominance of types II and III arthrogryposis in the present series is mainly related to a recruitment bias: patients seen in pediatric neurology units most often have non-limb body damage or central nervous system malformations or cognitive impairment. The etiology found most frequently in our series was therefore of a neurological type for $19.1 \%$ of the patients, with polymicrogyria predominating. However, the level of patients without any etiology remained high at $45 \%$, with several cases either still under discussion or awaiting the results of genetic studies.

Depending on the various series, the reason given most frequently was neurological impairment with a rate of $70-80 \%$, with the predominant type of impairment being that of the anterior horn of the spinal cord [7]. In the Vuopala series, three patients had problems with neuron migration and 15 had impairment of the anterior horn of the spinal cord [11]. Other authors, such as Banker, noted this characteristic, represented by impairment of the anterior horn of the spinal cord [12]. The present study brought out only two patients with this specific disorder, with the main hypothesis being that the patients with predominantly symmetrical distal arthrogryposis of an amyoplasia congenita type have not been studied in depth or seen at the various multidisciplinary consultations at the reference center. Historically, amyoplasia congenita is a subgroup of arthrogryposis multiplex congenita and accounts for $38-43 \%$ of patients. The diagnosis is mainly a clinical one grouping symmetrical damage to the joints of all four limbs, retained intellectual capacities and typical facial dysmorphology. Since the clinical symptoms of impairment of the anterior horn can be inconsistent, only a systematic electrophysiological study can identify or rule out this characteristic, as shown in the Gaitanis et al. study listing five

Table 1

Complementary assessments performed in connection with the clinical evaluation

\begin{tabular}{|c|c|c|c|c|c|c|c|c|c|c|c|}
\hline $\begin{array}{l}\text { Hall et al. all } \\
\text { classification }\end{array}$ & Number & $\mathrm{CPK}$ & $\begin{array}{l}\text { Electroneuromyogram } \\
\text { (ENMG) }\end{array}$ & $\begin{array}{l}\text { Muscle } \\
\text { biopsy (MB) }\end{array}$ & $\begin{array}{l}\text { Myasthenia } \\
\text { assessment }\end{array}$ & $\begin{array}{l}\text { Brain } \\
\text { MRI }\end{array}$ & $\begin{array}{l}\text { Spinal } \\
\text { cord MRI }\end{array}$ & $\begin{array}{l}\text { Cardiac } \\
\text { ultrasound }\end{array}$ & $\begin{array}{l}\text { Metabolic } \\
\text { assessment }\end{array}$ & $\begin{array}{l}\text { Genetic } \\
\text { investigation }\end{array}$ & $\begin{array}{l}\text { Definitive } \\
\text { diagnosis }\end{array}$ \\
\hline Type I & $8(19.1)$ & 6 & 7 & 2 & 2 & 5 & 3 & 4 & 3 & 5 & 2 \\
\hline Type II & $14(33.3)$ & 10 & 10 & 6 & 0 & 11 & 3 & 10 & 5 & 12 & 6 \\
\hline Type III & $20(47.6)$ & 9 & 11 & 6 & 6 & 17 & 10 & 15 & 7 & 16 & 15 \\
\hline Total & 42 & 25 & 28 & 14 & 8 & 33 & 16 & 29 & 15 & 33 & 23 \\
\hline
\end{tabular}

ENMG: electroneuromyogram; MB: muscle biopsy; MRI: magnetic resonance imaging; CPK: creatine phosphokinase. 
patients with symmetrical impairment of the anterior horn, sometimes segmental and especially cervical [13]. Among the types of impairment of the anterior horn sometimes associated with signs of arthrogryposis, we find infantile spinal muscular atrophy, X-linked spinal muscular atrophy, the Moebius syndrome, pontocerebellar hypoplasia and amyoplasia congenital.

The main neurological etiology in our population was polymicrogyria (four patients) particularly bilateral perisylvian polymicrogyria (three patients). No cause was found in the three patients, most particularly genetic. The perisylvian area is a major region since it is linked to the brain stem by the corticobulbar pathways and to the limbs by the corticospinal pathways and its integrity is essential for the normal development of the neurons downstream. Any malfunction may therefore be characterized by mixed clinical expression $[14,15]$. The origin of polymicrogyria may be either acquired (CMV infection, hypoxic-ischemic process, maternal intoxication, etc.) or genetic (microdeletion 22q11.2, variants in the $M E C P 2$ gene, genetic locus mapped by linkage analysis at $X q 28$, and recessive variants in PI4KA) [16,17]. Focal polymicrogyria, which is asymmetrical, is generally considered to be acquired. It appears at a later stage during the phase of cerebral organization [18]. Arthrogryposis secondary to impairment of the anterior horn of the spinal cord appears during the same embryological period as polymicrogyria (between 12 and 16 weeks' gestation).

The perisylvian region is a vulnerable zone but so is the anterior horn, especially in terms of vascularization. Hypoperfusion is likely to lead to secondary and associated spinal cord lesions. The other hypothesis is multisite impairment, of genetic origin, involving genes with a role in the differentiation of the neural tube [19].

We did not find any cases of congenital myopathy or muscular dystrophy in our population. In the various series, myopathic impairment corresponds to $20-30 \%$ of the etiologies [9].

On the other hand, there were four cases of genotyped distal arthrogryposis in this population, three of whom carried the mutation of the ECEL1 gene. Distal arthrogryposis, which is the most frequent cause of hereditary congenital contractures, is due, in $50 \%$ of cases, to mutations encoding striated muscle contractile proteins [20]. The best-known genes and those checked for most are genes that codify contractile proteins in muscle fibers (MYH2, MYH3, MYH8, MYBPC1, TPM2, TPM3, TNNI2, TNNT3, ACTA1, and NEB) or the genes involved in the homeostasis of calcium (RYR1) [20]. The involvement of this ECEL1 gene was essentially discovered in type II arthrogryposis, with the main clinical signs being distal arthrogryposis (particularly with camptodactyly), impairment of the knees resulting in hyperextension, atrophy and retained intelligence [8].

Three patients presented with arthrogryposis associated with limitation of fetal space (7.1\%). However, this high level should challenge the soundness of these etiological conclusions, which may have been too hasty. Finally, in a series including 2300 cases of arthrogryposis over 35 years, 33 cases $(1.3 \%)$ were due to an abnormality of the uterine structure [1].

The targeted complementary assessments performed allowed us to make a diagnosis for 23 patients (54.7\%). According to Hall's clinical classification, only two patients from group I ( $25 \%$ of type I), six in group II (43\%) and 15 from group III (75\%) were diagnosed.

In our population, the classification of arthrogryposis cases as reported in the literature and the heterogeneousness of conducting complementary assessments depending on the medical teams seem to indicate imperfect knowledge of this disease (Table 1) [21]. In this study, 33 brain MRIs and 16 spinal MRIs were performed and they were not systematically combined with cerebral MRI. Only two cases of medullary atrophy were found. Fedrizzi et al. showed that patients with arthrogryposis with brain abnormalities may have spinal cord injuries including atrophy of the anterior horn of the spinal cord [22]. Therefore, brain MRI should be systematically associated with spinal MRI for a complete etiological assessment. In this study, genetic assessment was not systematic (only for 33 patients). The genetic workup was a targeted analysis in association with next-generation sequencing for arthrogryposis. Genetic research should be targeted according to the clinical situation (type 5 distal arthrogryposis, polymicrogyria). However, there is not a systematic genotype-phenotype correlation and therefore a CGH array (because of the frequency of microdeletions) should be done or next-generation sequencing for arthrogryposis. Genetic consultation must therefore be systematic. This study clearly illustrates the problems with obtaining an accurate etiological diagnosis, in the absence of a standardized investigation protocol, both suitable and targeted. This results in genuine difficulties for, among other things, conducting genetic counseling. Filges \& Hall proposed guidelines for prenatal identification of MCC and fetal akinesia because in $73.8 \%$ of cases the diagnosis is missed prenatally. These recommendations are based on familial or maternal history and clinical signs detected by fetal ultrasound examination in all three trimesters. The aim is to detect early fetal movement disorder.

Accordingly, we have compiled a protocol for etiological investigation, aimed at standardizing professional practices, in accordance with recent documented data (Fig. 2).

Performing these complementary assessments must form part of coordinated treatment and multidisciplinary follow-up, to produce a standardized care pathway, to anticipate any situation involving a disability or multiple disabilities as effectively as possible.

We need to regularly check for restrictive respiratory syndrome and the symptoms of alveolar hypoventilation (clinical evaluation, respiratory functional exploration [RFE], capnography, or nocturnal polysomnography), depending on the etiology retained.

Nutritional status must be evaluated systematically, measuring weight, height, BMI (and brachial perimeter/skull perimeter up to the age of 4).

Cognitive functions and adaptation may need to be assessed, using standardized scales adapted to age and disability, in order to guide educational orientation.

Functional response to rehabilitation treatment must be evaluated at each consultation, guided by standardized functional scales.

We need to screen for osteoporosis by conducting patient interviews as well as performing phosphocalcium assessment and osteodensitometry.

Assessment of independence and quality of life should be systematic, assisted by standardized and validated scales.

\section{Conclusion}

Arthrogryposis is a sign suggesting multiple etiologies. The approach to this sign requires knowledge of embryology and the characteristics of fetal mobility. Fetal immobility syndrome is a common factor of the various etiologies encountered.

Prof. J.G. Hall's classification of arthrogryposis is used most frequently because it makes a clinical distinction by splitting the disease into three subgroups (types I, II and III). The etiologies are still quite varied, with the main ones being neurological, followed by myopathic. The impairment common to all these etiologies involves the central nervous system, sometimes associated with impairment of the anterior horn, indicating the complexity of this sign. Over 320 genes have been identified, allowing us to discover a wide variety of physiopathological mechanisms.

The diagnostic process must therefore be rigorous and targeted. Producing an etiological diagnosis may help establish both a vital 


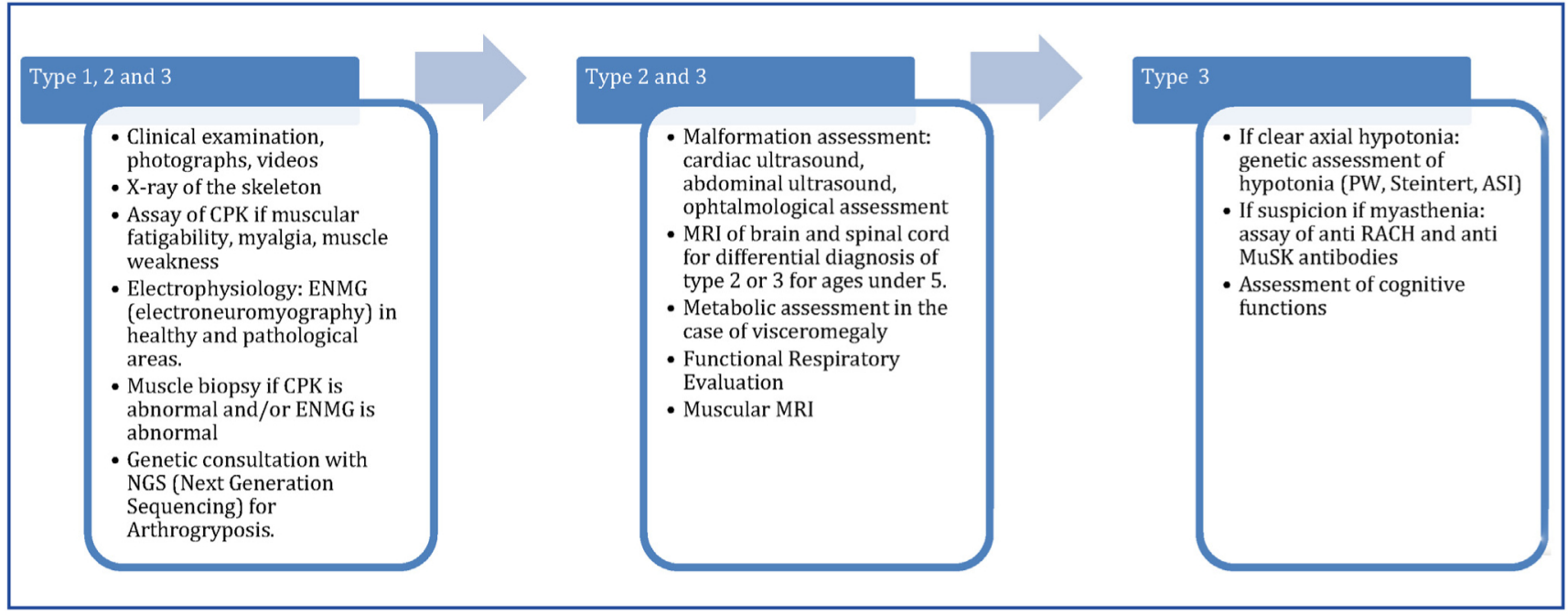

Fig. 2. Investigation protocol in arthrogryposis cases. ISA: infantile spinal muscular atrophy; PW: Prader-Willi; MRI: magnetic resonance imaging; CPK: creatinine phosphokinase; ENMG: electroneuromyogram. This protocol shows the different investigations to be carried out for an etiological diagnosis according to the clinical type of arthrogryposis (I, II, III), in order to standardize professional practices.

prognosis and a functional one. This may also be a critical issue in genetic counseling.

Producing a protocol for graduated paraclinical examinations, as described above, should standardize the diagnostic process as well as its efficiency.

Treatment remains multidisciplinary and coordinated in order to detect any impact on motor, respiratory, or nutritional functions, and possibly on quality of life. Anticipation is essential in order to limit any situation that might lead to disability.

\section{Funding}

No financial aid was provided for this research.

\section{Disclosure of interest}

The authors declare that they have no competing interest.

\section{References}

[1] Hall JG. Uterine structural anomalies and arthrogryposis-death of an urban legend. Am J Med Genet Part A 2013;161:82-8.

[2] Lowry RB, Sibbald B, Bedard T, et al. Prevalence of multiple congenital contractures including arthrogryposis multiplex congenita in Alberta, Canada, and a strategy for classification and coding. Birth Defects Res A Clin Mol Teratol 2010;88:1057-61.

[3] Filges I, Hall JG. Failure to identify antenatal multiple congenital contractures and fetal akinesia - proposal of guidelines to improve diagnosis. Prenat Diagn 2013;33:61-74.

[4] Hall JG. Arthrogryposis multiplex congenital: etiology, genetics, classification, diagnostic approach and general aspects. J Pediatr Orthop B 1997;6:159-66.

[5] Hall JG. Arthrogryposes (multiple congenital contractures). In: Rimoin DL, Pyeritz RE, Korf BR, editors. Emery and Rimoin's principle and practice of medical genetics. Sixth Ed., New York: Churchill Livingstone; 2013. p. 1-161 [Chapter 161].
[6] Hall JG, Kiefer J. Arthrogryposis as a syndrome: gene ontology analysis. Mol Syndromol 2016;7:101-9.

[7] Kalampokas E, Kalampokas T, Sofoudis C, et al. Diagnosing arthrogryposis multiplex congenita: a review. ISRN Obstet Gynecol 2012;2012. http: dx.doi.org/10.5402/2012/264918 [264918].

[8] Hall JG. Arthrogryposis (multiple congenital contractures): diagnostic approach to etiology, classification, genetics and general principles. Eur J Med Genet 2014;57:464-72.

[9] Bamshad M, Van Heest AE, Pleasure D, et al. A review and update. J Bone Joint Surg Am 2009;91(Suppl. 4):40-6.

[10] Hoff JM, Loane M, Gilhus NE, et al. Arthrogryposis multiplexa congenita: an epidemiologic study of nearly 9 million births in 24 EUROCAT registers. Eur J Obstet Gynecol Reprod Biol 2011;159:347-50.

[11] Vuopala K, Leisti J, Herva R. Lethal arthrogryposis in Finland clinical-pathological study of 83 cases during thirteen years. Neuropediatrics 1994;25:308-15.

[12] Banker BQ. Arthrogryposis multiplex congenita: spectrum of pathologic changes. Hum Pathol 1986;17:656-72.

[13] Gaitanis JN, McMillan HJ, Wu A, et al. Electrophysiologic Evidence for anterior horn cell disease in amyoplasia. Pediatr Neurol 2010;43:142-7.

[14] Poduri A, Chitsazzadeh V, D’Arrigo S, et al. The syndrome of perisylvian polymicrogyria with congenital arthrogryposis. Brain Dev 2010:32:550-5.

[15] Marret S, Charollais A, Vanhulle C, et al. Pathologie périnatale acquise du fœtus et nouveau-né prématuré et à terme. In: Chabrol B, Mancini J, Dulac O, et al., editors. Neurologie pédiatrique. $3^{\mathrm{e}}$ édition, Paris: Médecine Sciences Flammarion; 2010. p. 210

[16] Villard L, Nguyen K, Cardoso C, et al. A locus for bilateral perisylvian polymicrogyria maps to Xq28. Am J Hum Genet 2002;70:1003-8.

[17] Pagnamenta T, Howard MF, Wisniewski E, et al. U. Germline recessive mutations in PI4KA are associated with perisylvian polymicrogyria, cerebellar hypoplasia and arthrogryposis. Hum Mol Genet 2015;24:3732-41.

[18] De Coene A, Van Coster R, Verhelst H. Perisylvian polymicrogyria, infantile spasms and arthrogryposis: the severe end of the spectrum of congenital bilateral perisylvian polymicrogyria. Eur J Paediatr Neurol 2010;14:270-3.

[19] Clark M, Pitt M, Neville BG. Lower motor neuron involvement in perisylvian polymicrogyria. Dev Med Child Neurol 2006;48:842-6.

[20] McMillin MJ, Below JE, Shively KM, et al. Mutations in ECEL1 cause distal arthrogryposis type 5D. Am J Hum Genet 2013;92:150-6.

[21] Wong V. Original article The spectrum of arthrogryposis in 33 Chinese children. Brain Dev 1997;19:187-96.

[22] Fedrizzi E, Botteon G, Inverno M, et al. Neurogenic arthrogryposis multiplex congenita: clinical and MRI findings. Pediatr Neurol 1993;9:343-8. 